\title{
Single stage gamma knife radiosurgical treatment of large AVMs
}

\author{
Roberto C. Heros
}

Received: 9 November 2011 / Accepted: 25 November 2011 /Published online: 16 December 2011

(C) Springer-Verlag 2011

The authors of this important paper treated a remarkable 564 patients with large cerebral AVMs with single-stage gamma knife radiosurgery between 1986 and 2007. They define large AVMs as those having a volume of $10 \mathrm{~cm}^{3}$ or more. From this group, they obtained "complete radiologic followup," which I presume to be catheter angiography, in 400 patients, and they had "useful clinical follow-up information" in a total of 492 patients; those constitute the material analyzed in their paper. From my perspective, the two important points that the authors make with their analysis are that large AVMs can be treated with modest effectiveness and relative safety with single-stage gamma knife radiosurgery, and that the results of such treatment have improved substantially with improvements in technique and imaging.

To address the second point, the authors divided the patients into three groups depending on their treatment time period. The first group of 157 patients was treated between 1986 and 1993 with what the authors call a "simplistic" technique based only on angiography and using a median of two isocenters covering only 45 to $70 \%$ of the AVM. The second group of 225 patients was treated between 1994 and 2000 with a more sophisticated plan that included a median of five isocenters covering 64 to $95 \%$ of the AVM. The third group of 182 patients has been treated since 2000 with a median of seven isocenters covering 62 to $94 \%$ of the AVM, and importantly, incorporating MRI combined with angiography in the treatment plan. The rates of complete obliteration during the three periods were $27 \%, 30 \%$ and $52 \%$, respectively. The rates of "radiation injury" were $16 \%$, $12 \%$ and $16 \%$, respectively. In other words, it is clear that

R. C. Heros $(\bowtie)$

Department of Neurosurgery, University of Miami,

Miami, FL, USA

e-mail: RHeros@med.miami.edu the authors obtained a much higher rate of complete obliteration with modern techniques that included MRI as well as angiography for planning the target, and they achieved this increased effectiveness without a concommitent increase in morbidity. In fact, the rate of serious permanent morbidity was lower during the third period.

The authors provide an extensive amount of data to support the concept that single-session radiosurgery is effective for the treatment of large AVMs. They produce a very useful breakdown of these AVMs in three different groups depending on size. The obliteration rate during the third period where modern techniques were used was $76 \%$ for the smallest AVMs (10 to $12 \mathrm{~cm}^{3}$ ); the intermediate group of 12 to $20 \mathrm{~cm}^{3}$ had an obliteration rate of $52 \%$, but the obliteration rate, even using the most modern technique, was only $29 \%$ for the large AVMs of more than $20 \mathrm{~cm}^{3}$. It is important to keep in mind that these obliteration rates are at 4 years and that these patients remain at significant risk of re-bleeding before obliteration. In fact, after treatment the rate of bleeding for unruptured AVMs was 3.6\% per year, which appears to be higher than the rate of hemorrhage for unruptured AVMs before treatment. AVMs that had bled before treatment re-bled at a rate of $6.6 \%$ per year, which approximates the rate of bleeding before treatment for these patients. It is of course also important to consider the rate of radiation injury after treatment, but I could not find their specific percentages of these complications in relation to size, although from the general discussion, the authors seem to indicate that that rate is not significantly higher on the large AVMs in their particular series. With these considerations, I am left to conclude that single-session radiosurgery is ineffective for very large AVMs, but could be considered for those of intermediate size when other treatment options such as embolization followed by surgical excision appear to be excessively risky. Clearly, with an obliteration rate of $76 \%$, 
single-session radiosurgery appears to be an attractive alternative for the smallest group of AVMs measuring $10-12 \mathrm{~cm}^{3}$ in volume.

In an important sub-analysis of their large series, the authors conclude that embolization prior to radiosurgery worsens the overall results. In all three of their time periods, embolization led to a lower obliteration rate, and in the overall series, the obliteration rate of those AVMs that had been previously partially embolized was only $26 \%$ as compared to $38 \%$ in those that had no prior embolization. Even with modern techniques, during their third period of time, the obliteration rate of those AVMs that had previously been embolized was $36 \%$ as compared to $61 \%$ for those that had no prior embolization. With these data, the authors confirmed the findings of several other groups to the effect that partial embolization before radiosurgery leads to a lower obliteration rate without significantly decreasing the hemorrhage rate after radiosurgery before complete obliteration. We have reviewed this issue extensively and have come to the same conclusion that generally there is no advantage to embolization before radiosurgery, particularly considering the fact that embolization itself carries a significant morbidity as many authors, including ourselves, have discussed in the past $[1,2]$. Of course, there are some particular circumstances, such as repeated hemorrhages, high flow intranidal fistulas, venous outflow constriction and perhaps intranidal aneurysms, which may make pre-radiosurgical embolization a desirable option.

In summary, the authors have provided us with an insightful historical review of the results of single-session radiosurgery for large AVMs. They emphasize the fact that this is a historical review to serve as a baseline of comparison with more modern treatment paradigms. They tell us that currently they are treating most very large AVMs with "staged" rather than single-session radiosurgery. For smaller AVMs, their series confirms the value of single-session radiosurgery but does not, of course, prove that radiosurgery is the treatment of choice for those AVMs. Seven percent of the patients treated in this series had Spetzler-Martin grade II AVMs, and it has been well established that experienced vascular neurosurgeons can achieve complete excision of essentially all of these AVMs with minimal morbidity, which makes it difficult to justify the use of radiosurgery, except in very special circumstances, for grade II AVMs [3]. Surgical morbidity is slightly higher but still generally below $10 \%$ in Spetzler-Martin grade III AVMs; however, as has been pointed out by many, this is a very heterogeneous group, and the choice of surgery, usually preceded by embolization vs. radiosurgery, is one that must be considered individually on a case-by-case basis depending upon many factors, including the experience of the operator. I still feel that there aren't any satisfactory treatments for grade V AVMs at the present time, although multi-modality treatment, including staged radiosurgery, could be considered for symptomatic patients that have bled repeatedly. With Spetzler-Martin grade IV AVMs, staged radiosurgery may become a competitive form of treatment, although with proper selection many of these lesions can be surgically excised after embolization with reasonable results [3].

Conflicts of interest None.

\section{References}

1. Baskaya MK, Heros RC (2006) Indications for and complications of embolization of cerebral arteriovenous malformations. J Neurosurg 104:183-187

2. Heros RC (2004) Embolization of arteriovenous malformations. J Neurosurg 100:807-809

3. Heros RC, Korosue K, Diebold PM (1990) Surgical excision of cerebral arteriovenous malformations: Late results. Neurosurgery 26:570-577 